\title{
Raper Ka Band-pass Filter Based on SIW Technology for Wireless Communications
}

\author{
Mehdi Damou $^{1}$, Yassine Benallou ${ }^{1}$, Mohammed Chetioui ${ }^{2}$, Abdelhakim Boudkhil ${ }^{2}$, \\ and Redouane Berber ${ }^{1}$ \\ ${ }^{1}$ Dr. Tahar Moulay University of Saida, Saida, Algeria \\ ${ }^{2}$ Abu Bakr Belkaid University of Tlemcen, Tlemcen, Algeria
}

\begin{abstract}
The paper proposes a new third-order Chebyshev bandpass filter based on the substrate integrated waveguide (SIW) manufacturing technology using an inductive iris and a defected ground structure (DGS) station to resonate in the Ka frequency band, intended for wireless communication applications. All steps that are necessary for designing such a filter have been described in detail based on specific analytical equations harnessed to calculate the different synthesizable parameters of the proposed band-pass filter design, such as the coupling matrix, quality coefficients and initial geometric dimensions. The filter's ideal frequency response is extracted from an equivalent circuit employing localized elements developed with the use of Design Microwave Office Software. Otherwise, HFSS is employed to set the initial parameters of the proposed topology that will not meet the target specifications defined previously. Accordingly, optimization procedures are necessary for different SIW band-pass filter parameters to reach a high frequency response for the proposed design. The detailed results presented show high efficiency of the SIW technology that offers good performance with lower filter volumes. Two topologies have been developed and then optimized to demonstrate the usefulness of EM software.
\end{abstract}

Keywords-coupling matrix, DGS, filters, Ka band, SIW.

\section{Introduction}

Operational frequency bands used in satellite wireless systems have become highly saturated these days, especially due to huge demand created by multimedia applications. This requires that the operational frequency range be broadened by considering new design techniques and technologies for different passive and active microwave devices. Accordingly, a thorough investigation has been performed to study the behavior of superconducting front-end planar devices at the Ka band $(26-40 \mathrm{GHz})$ in order to expand the additional range and to meet the future requirements of wireless communications [1]. In fact, with all the prospects associated with this considerable frequency band, it is always desirable to design simple, robust and reliable components, characterized by reduced weight, energy consumption and by low cost. Accordingly, this study investigates primarily the modern technologies relied upon for developing integrated filters operating in the Ka band, notably the SIW technology, in order to demonstrate its high integrability and to achieve good performance parameters.

Traditionally, waveguides were used in designing highperformance filters that claimed a complicated transition to integrated planar devices taking into account their bulky size. The straight forward solution is to combine a rectangular waveguide into the microstrip structure, forming the so-called substrate-integrated waveguide [2] offering good quality factor $\mathrm{Q}$ at the input due to the dielectric filling caused by volume reduction [3]. SIW side walls are commonly formed by using metallic via-holes or post walls [4]. Furthermore, many manufacturing techniques may be combined with the substrate integrated waveguide technology to implement different filter topologies. These include, for instance, the DGS technique that is an emerging method relied upon to enhance such filter parameters as narrow operating bandwidth or high weight [5].

This paper presents two design topologies of a novel third pole band-pass filter based on the SIW technology to operate at the Ka band. The first design uses a simple cavity structure. The other, however, employs a new SIW-DGS structure to eliminate losses by avoiding disturbance close to the resonance point and by suppressing higher mode harmonics, thus mastering mutual coupling.

The paper is organized as follows. Section 1 presents the main synthesis steps and coupling matrix calculations for the SIW filter. Section 2 describes, in detail, the process of synthesizing, simulating and optimizing the three pole SIW filter. Section 3 illustrates the design principles of the proposed filter and smiling of the admittance inverter based on perforated, and Section 4 compares the two topologies of the three pole SIW filter.

\section{Substrate Integrated Wave Guide Filters}

SIW filters with inductive shunt coupling may be designed easily using an air-filled rectangular waveguide. The filter 
may be formed by either a shunt inductive post or an iris inductive shunt aperture, as reported in [6]. As indicated in Fig. 1, the first example of the developed SIW bandpass filter model employs the iris inductive shunt. Different resonances are caused due to inductive shunt coupling, in addition to two microstrip-to-SIW transitions at the input and the output. The smallest inductive shunts facilitate input/output coupling, while the biggest ones provide interresonator coupling [1]. The filter design procedures are similar to those of an air-filled waveguide filter based on the coupling-matrix technique. Finally, properties of the modeled SIW filter make it possible to manufacture low profile and low-cost microwave filters.

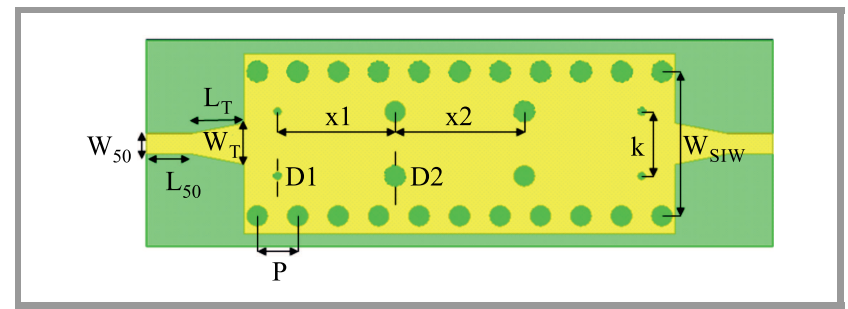

Fig. 1. First SIW band-pass filter model with the iris inductive shunt.

\section{Formation of the Coupling Matrix from the Low Pass Prototype}

The transmission coefficient of a low pass prototype filter network, defined as the ratio of the transmitted power to the available power, may be represented as [7]:

$$
\left|S_{21}(s)\right|_{s=\mathrm{j} \omega}^{2}=\frac{1}{1+\varepsilon^{2}\left|K_{N}(s)\right|_{s=\mathrm{j} \omega}^{2}}
$$

where $\varepsilon$ is related to the band-pass ripple and the band-pass return loss $R_{L}$ via:

$$
\varepsilon=\frac{1}{\sqrt{10^{\frac{R_{L}}{10}}-1}} .
$$

$K_{N}(s)$ is the characteristic function of degree $N$ and has the following form:

$$
\left\{\begin{array}{l}
K_{N}(\mathrm{j} \omega) \cosh \left(\sum_{n=1}^{N} \cosh h^{-1}\left(x_{n}\right)\right) \\
x_{n}=\frac{\omega-\frac{1}{\omega_{n}}}{1-\frac{\omega}{\omega_{n}}}
\end{array}\right.
$$

where $s_{n}=\mathrm{j} \omega_{n}$ is the location of the $n$-th transmission zero in the complex $s$ plane. Since the rational polynomial $K_{N}(s)$ represents the ratio of polynomials $F(s)$ and $P(s)$, it is more appropriate to represent $K_{N}(s)$ in the following form:

$$
K_{N}(s)=\frac{F(s)}{\frac{P(s)}{\varepsilon}} .
$$

Polynomials $F(s)$ and $P(s)$ are formed by the zeros of reflection and transmission, respectively, and are assumed to be known with their highest coefficients as unity. Using the conservation of energy formula for a lossless filter, the polynomial $E(s)$ is determined as:

$$
|E(s)|^{2}=|F(s)|^{2}+\frac{|P(s)|^{2}}{\varepsilon^{2}} .
$$

The coupling values $M_{i j}(i=1,2, \ldots, N$ and $j=1,2, \ldots, N)$ fully define the filter. Using the Kirchhoff's voltage law, the coupling matrix is derived via an impedance matrix from a set of loop equations.

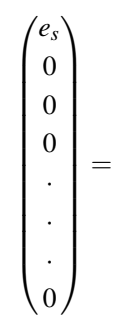

$$
\left(\begin{array}{cccccc}
s+R_{s} & -\mathrm{j} \omega M_{12} & -\mathrm{j} \omega M_{13} & \ldots & \cdot & -\mathrm{j} \omega M_{1 N} \\
-\mathrm{j} \omega M_{12} & s & -\mathrm{j} \omega M_{23} & \ldots & \cdot & \cdot \\
-\mathrm{j} \omega M_{13} & -\mathrm{j} \omega M_{23} & s & \ldots & \cdot & \cdot \\
\cdot & \cdot & \cdot & \ldots & \cdot & \cdot \\
\cdot & \cdot & \cdot & \ldots & \cdot & \cdot \\
\cdot & \cdot & \cdot & \ldots & \cdot & \cdot \\
\cdot \mathrm{j} \omega M_{1 N} & \cdot & \ldots & -\mathrm{j} \omega M_{N, N-1} & s+R_{L} &
\end{array}\right)\left(\begin{array}{c}
i_{1} \\
i_{2} \\
i_{3} \\
0 \\
\cdot \\
\cdot \\
\cdot \\
i_{N}
\end{array}\right)
$$

The loop analysis of the filter yields the following result:

$$
[e]=[A][i] .
$$

For $L=L_{1} \ldots=L_{i}[\mathrm{H}], C=C_{1} \ldots C_{i}[\mathrm{~F}]$, and $s=\mathrm{j} \omega$ and the two-port scattering parameters, the transfer and reflection functions are given by [3]:

$$
\begin{aligned}
& S_{21}=-2 \mathrm{j} \sqrt{R_{S} R_{L}}[A]_{(n, 1)}^{-1} \\
& S_{11}=1+2 \mathrm{j} R_{S}[A]_{(1,1)}^{-1} .
\end{aligned}
$$

The general matrix $A$ comprising coupling coefficients $m_{i j}$ and external quality factors $q_{i, e x t}$ is presented in [4] as:

$$
\begin{aligned}
& {[A]=\left[\begin{array}{ccc}
\frac{1}{q_{e 1}} & 0 & 0 \\
0 & 0 & 0 \\
\vdots & \ddots & \vdots \\
0 & \ldots & \frac{1}{q_{e n}}
\end{array}\right]} \\
& +p\left[\begin{array}{cccc}
1 & 0 & \ldots & 0 \\
0 & 1 & \ldots & 0 \\
\vdots & \vdots & \ldots & \vdots \\
0 & 0 & \ldots & 1
\end{array}\right]-\mathrm{j}\left[\begin{array}{cccc}
m_{1,1} & m_{1,2} & \ldots & m_{1, n} \\
m_{2,1} & m_{2,2} & \ldots & m_{2, n} \\
\vdots & \vdots & \ldots & \vdots \\
m_{n, 1} & m_{n, 2} & \ldots & m_{n, n}
\end{array}\right],
\end{aligned}
$$

$$
p=\mathrm{j} \frac{1}{\mathrm{FBW}}\left(\frac{\omega}{\omega_{0}}-\frac{\omega_{0}}{\omega}\right),
$$




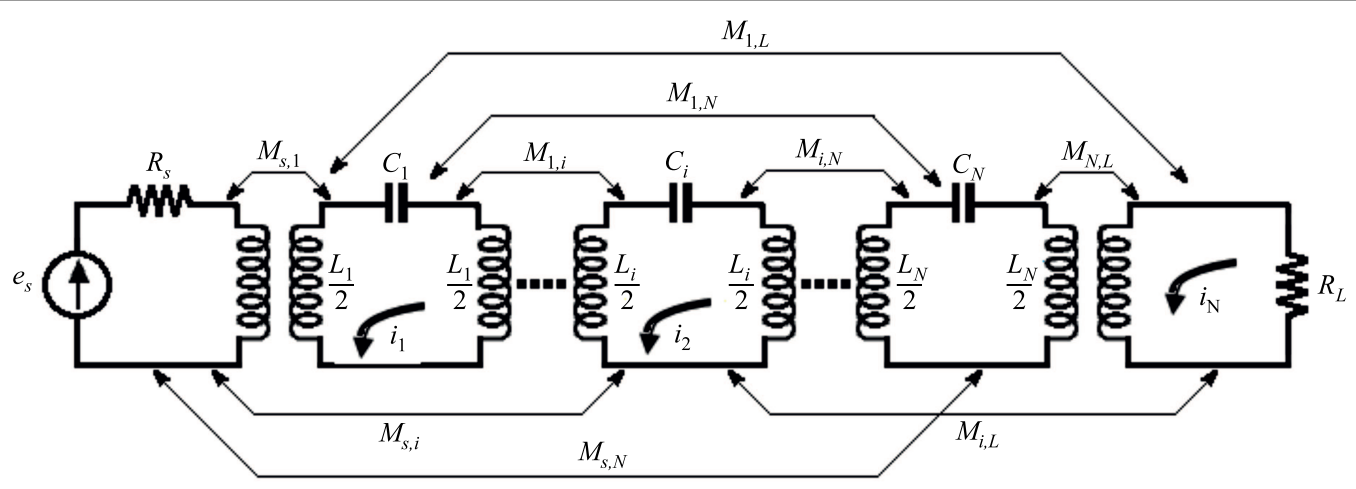

Fig. 2. Generalized model of a cross-coupled resonator filter.

where $p$ is the complex lowpass frequency, $\omega_{0}$ is the center frequency of the filter, FBW is the fractional bandwidth of the filter, $q_{i, \text { ext }}(i=1, \ldots, n)$ is the scaled external quality factors of the resonator $i$, and $m_{i j}$ is the normalized coupling coefficients between the resonator $i$ and $j$ [8].

\section{SIW Filter Design Using Cross Coupled Resonators}

For demonstration purposes, a highly selective third-pole SIW filter with the configuration shown in Fig. 2 has been designed. A cross coupled model is given as an example to illustrate the proposed solution. The target specifications of the SIW band-pass filter are shown in Table 1.

Table 1

Target specifications of the SIW band-pass filter

\begin{tabular}{|l|c|}
\hline Center frequency & $f_{o}=28.86 \mathrm{GHz}$ \\
\hline \hline Bandwidth & $\mathrm{BW}=1050 \mathrm{MHz}(\mathrm{FBW}=3.63 \%)$ \\
\hline Bandpass ripples & $L_{A R}=0.0431 \mathrm{~dB}$ \\
\hline $\begin{array}{l}\text { Bandpass return } \\
\text { loss }\end{array}$ & $R_{L}=20 \mathrm{~dB}$ \\
\hline $\begin{array}{l}\text { Stopband rejection } \\
\text { level }\end{array}$ & $>40 \mathrm{~dB}$ \\
\hline
\end{tabular}

The element values of the lowpass prototype filter are found to be $g_{1}=g_{3}=0.8516, g_{2}=1.1032$. The design parameters, i.e. the de-normalized external quality factors, can be determined by means of the following formulas [9]:

$$
\begin{gathered}
Q_{e S}=\frac{g_{0} g_{1}}{\mathrm{FBW}}, \quad Q_{e L}=\frac{g_{n} g_{n+1}}{\mathrm{FBW}}, \\
M_{i, i+1}=\frac{\mathrm{FBW}}{\sqrt{g_{i} g_{i+1}}}, \quad i=1 \text { to } n-1, \\
m_{i, i+1}=\frac{M_{i, i+1}}{\mathrm{FBW}}, \quad i=1, \ldots, n .
\end{gathered}
$$

From Eq. (10), in the first step of the design, the lowpass prototype filter elements with the following ideal coupling matrix are evaluated:

$$
\begin{gathered}
M=\left[\begin{array}{ccc}
0 & 0.0375 & 0 \\
0.0375 & 0 & 0.0375 \\
0 & 0.0375 & 0
\end{array}\right] . \\
M=Q_{\text {ext_e }}=Q_{\text {ext_s }}=23.4068 .
\end{gathered}
$$

Capacitance $C_{0}$ and the inductance $L_{0}$ are derived from:

$$
\begin{gathered}
C_{0}=\frac{Q_{e 1}}{w_{0} \times Z}=2.5816 \mathrm{pF}, \\
L_{0}=\frac{Q_{e 1}}{w_{0} \times Q_{e 1}}=0.0118 \mathrm{nH} .
\end{gathered}
$$

The series impedances $Z_{12}, Z_{23}, Z_{34}$ are:

$$
Z_{i, i+1}=\frac{Z}{M_{i-1, i} \times Q_{e 1}} .
$$

The series impedances are: $Z_{01}=50 \Omega, Z_{12}=56.91 \Omega$, $Z_{23}=56.91 \Omega$.

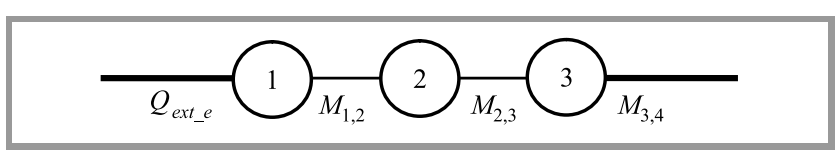

Fig. 3. Generalized model for SIW coupled resonator filter.

Figure 3 shows the corresponding coupling/routing diagram with SIW resonators used to implement the filtering methods that is characteristic of the SIW technology. $M_{i, i+1}$ indicates the direct coupling sequence.

Figure 4 shows an equivalent circuit of the BPF. This circuit consists of three parallel LCR resonators which are separated by wavelength microstrip lines. As shown in Fig. 4, the transmission line $\theta= \pm 90^{\circ}$ at the center frequency and $w_{0}=\frac{1}{\sqrt{L}}$ are employed to represents the coupling coefficient. This circuit model does account for the loss effect. The RLC microstrip transmission line unit section is simulated using Microwave Office (AWR) Software. The proposed filter's ideal frequency response of the equivalent circuit is presented in Fig. 5, which provides a return loss coefficient of less than $25 \mathrm{~dB}$ for a bandpass of $1.05 \mathrm{GHz}$ at the center frequency of $28.86 \mathrm{GHz}$. 


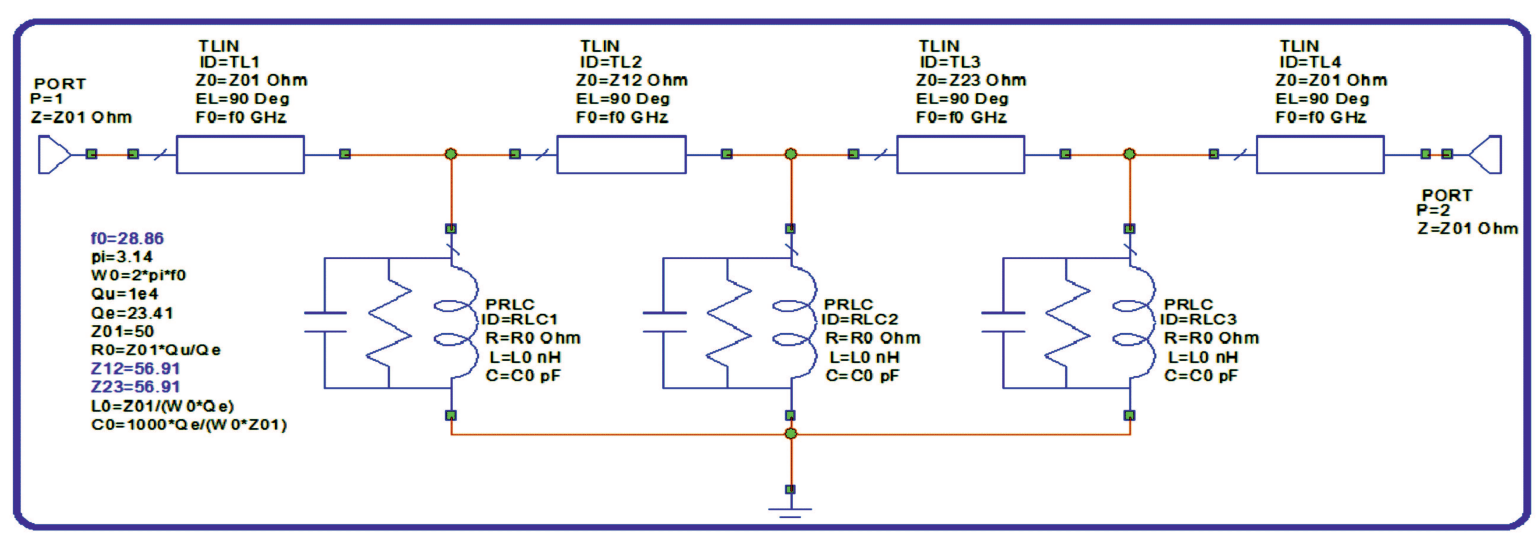

Fig. 4. Coarse model of the third order SIW band-pass filter.

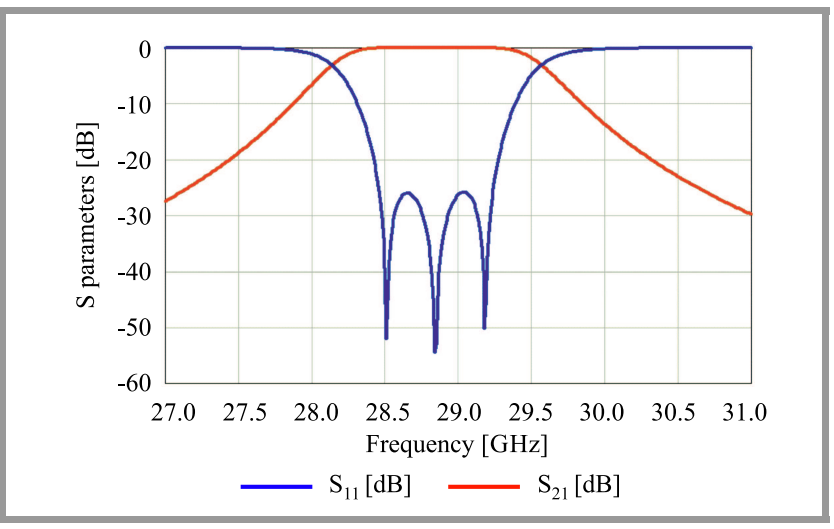

Fig. 5. Ideal response curve of the third order SIW to a band-pass filter based on the CM technique.

\section{Simulation of Third Order Band-Pass SIW Filter}

In this section, a third order iris SIW band-pass filter is simulated. In the prototype fabrication process, the Arlon Di-Clad 880 substrate with a thickness of $0.25 \mathrm{~mm}$, a dielectric constant of 2.2 and a loss tangent of 0.009 was used. For the desired resonance frequency of $28.86 \mathrm{GHz}$, diameter $d$ of the metal sights is $0.7 \mathrm{~mm}$, with pitch $P$ of $1.04 \mathrm{~mm}$ to prevent radiation leakage [10]:

$$
\left(\begin{array}{l}
\lambda_{c}=\frac{c}{f_{101} \sqrt{\varepsilon_{r}}}=\frac{3 \cdot 10^{8}}{28.86 \cdot 10^{9} \sqrt{2.2}} \approx 7 \mathrm{~mm} \\
\frac{d}{\lambda_{c}} \approx 0.1 \Rightarrow d=0.7 \mathrm{~mm} \\
\frac{d}{P} \approx 0.67 \Rightarrow P=1.04 \mathrm{~mm} .
\end{array}\right.
$$

Length Leq and width Weq are equal to $5 \mathrm{~mm}$ for a single SIW square cavity, as calculated in:

$$
\begin{gathered}
f_{101}=\frac{c}{2 \pi \sqrt{\varepsilon_{r}}} \sqrt{\left(\frac{\pi}{W e q}\right)^{2}+\left(\frac{\pi}{L e q}\right)^{2}}=28.86 \mathrm{GHz} \\
W e q=\text { Leq }=5 \mathrm{~mm} .
\end{gathered}
$$

$W_{\text {SIW }}$ spacing between the two rows of holes is a relevant physical parameter that is necessary for designing a SIW structure. It may be determined from empirical equations as a function of the equivalent width Weq of the rectangular waveguide, offering the same characteristics in the fundamental mode for the same height and the same dielectric constant:

$$
\begin{gathered}
W_{\mathrm{SIW}}=W e q+\frac{d^{2}}{0.95 P} \approx 5.5 \mathrm{~mm} \\
W_{\mathrm{SIW}}=L=5.5 \mathrm{~mm} \text { (square cavity) } \\
W_{t}=0.4\left(W_{\text {SIW }}-d\right) \approx 7.304 \mathrm{~mm}, \\
\left(\begin{array}{l}
\frac{\lambda}{2} \leq L_{t} \leq \lambda \\
\lambda=\frac{c}{f_{0} \sqrt{\varepsilon_{r}}} \\
L_{t} \approx 13.12 \mathrm{~mm} .
\end{array}\right.
\end{gathered}
$$

The preliminary dimensions of the third-order band-pass filter structure are presented in Table 2, and its topology is shown in Fig. 6. The three resonators are coupled together using an inductive iris, while the two end resonators are coupled to excite and load by $50 \Omega$ microstrip line.

Figure 7 illustrates a very low frequency response when simulating the structure's initial geometric parameters

Table 2

Preliminary dimensions of the third order band-pass filter

\begin{tabular}{|c|c|c|c|}
\hline $\begin{array}{c}\text { Parameters } \\
\text { group }\end{array}$ & Type & Descriptor & $\begin{array}{c}\text { Size } \\
{[\mathrm{mm}]}\end{array}$ \\
\hline \hline \multirow{2}{*}{ Taper } & Width & $W_{\text {Taper }}$ & 1.92 \\
\cline { 2 - 4 } & Length & $L_{\text {Taper }}$ & 4.00 \\
\hline \multirow{3}{*}{ SIW guide } & Width & $W_{\text {SIW }}$ & 5.50 \\
\cline { 2 - 4 } & Via & $P$ & 1.04 \\
\cline { 2 - 4 } & Diameter & $D_{1}$ & 0.70 \\
\hline \multirow{3}{*}{ Resonator } & Width & $x_{1}$ & 4.50 \\
\cline { 2 - 4 } & Width & $x_{2}$ & 4.50 \\
\cline { 2 - 4 } & Width & $D_{2}$ & 0.15 \\
\cline { 2 - 4 } & Length & $k$ & 2.40 \\
\hline
\end{tabular}




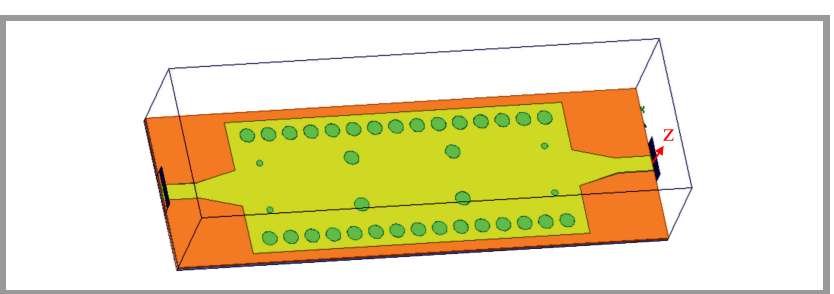

Fig. 6. 3D view of the proposed filter.

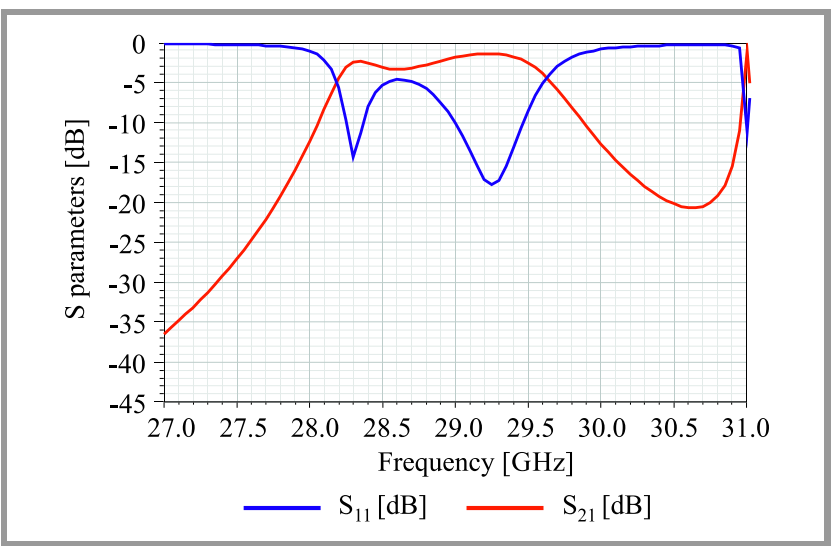

Fig. 7. S parameters of the proposed SIW band-pass filter with initial geometric parameters.

which provide a very poor reflection and transmission coefficients of $5 \mathrm{~dB}$ and $2 \mathrm{~dB}$, respectively, for a passband greater than $1.45 \mathrm{GHz}$ in the $28.20-29.65 \mathrm{GHz}$ band. Accordingly, the structure is then optimized using HFSS software to improve the filter response.

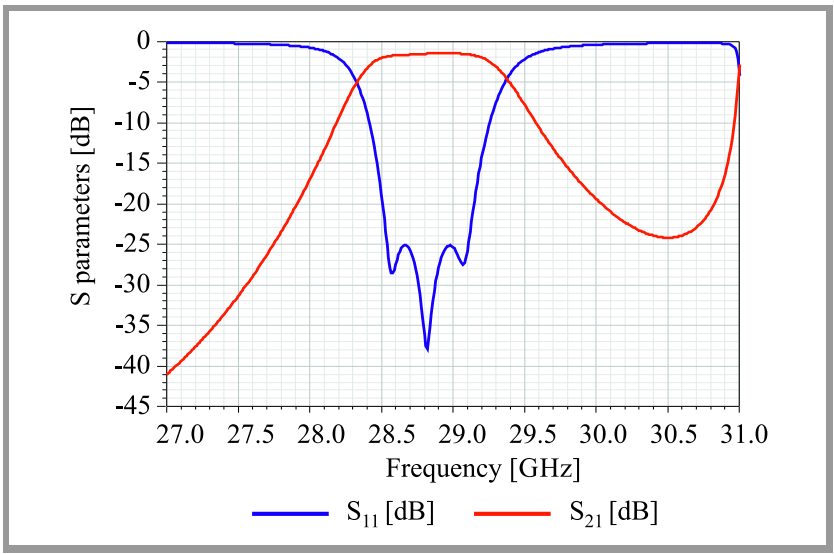

Fig. 8. S parameters of the optimized SIW band-pass filter.

Figure 8 shows a high frequency response for the optimized structure. The reflection coefficient is less than $25 \mathrm{~dB}$ and the transmission coefficient is approximately $1 \mathrm{~dB}$ for the resonance frequency of $28.82 \mathrm{GHz}$ and a passband of $1.05 \mathrm{GHz}$. Table 3 presents, in detail, the different dimensional changes introduced based on optimization procedures. Optimized dimensions of the 3rd order SIW bandpass filter structure are presented in Table 3 .
Table 3

Optimized dimensions of the filter

\begin{tabular}{|c|c|c|c|}
\hline Parameter & Size $[\mathrm{mm}]$ & Parameter & Size $[\mathrm{mm}]$ \\
\hline \hline$W_{50}$ & 0.8 & $l_{50}$ & 2.00 \\
\hline$W_{t}$ & 1.6 & $L_{t}$ & 4.00 \\
\hline$W_{\text {SIW }}$ & 5.6 & $a_{1}$ & 4.95 \\
\hline$d_{1}$ & 0.80 & $a_{1}$ & 4.50 \\
\hline$d_{2}$ & 0.15 & $k$ & 2.4 \\
\hline
\end{tabular}

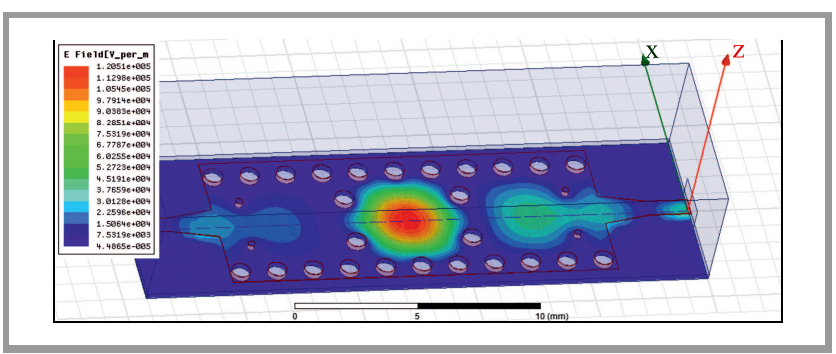

Fig. 9. Simulated E field distribution.

Figure 9 presents the simulated field layout of the proposed SIW band-pass filter for the TE101 propagation mode, at the center frequency with two circular propagating zones in a single SIW filter cavity.

\section{SIW Structure Variation Effects}

As shown in Fig. 10, after optimizing the iris inductive shunt filter with the use of the HFSS simulator, the frequency response becomes very close to the ideal specification with a passband of $1.045 \mathrm{GHz}$, center frequency of approx. $28.85 \mathrm{GHz}$, and reflection losses of less than $25 \mathrm{~dB}$. The curves presented in Fig. 11 demonstrate that variations of the opening diameter influence the center frequency inversely to the WSIW width variation effect. In fact, an increase in the diameter causes right shifting, while a decrease in the diameter causes left shifting. As a result, the

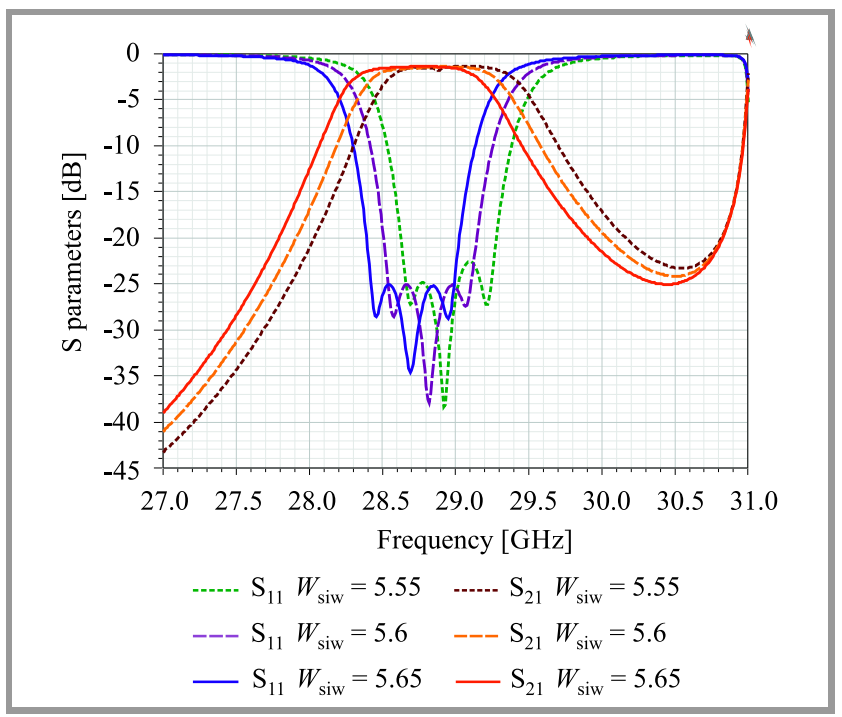

Fig. 10. $W_{\text {SIW }}$ spacing variation effect. 


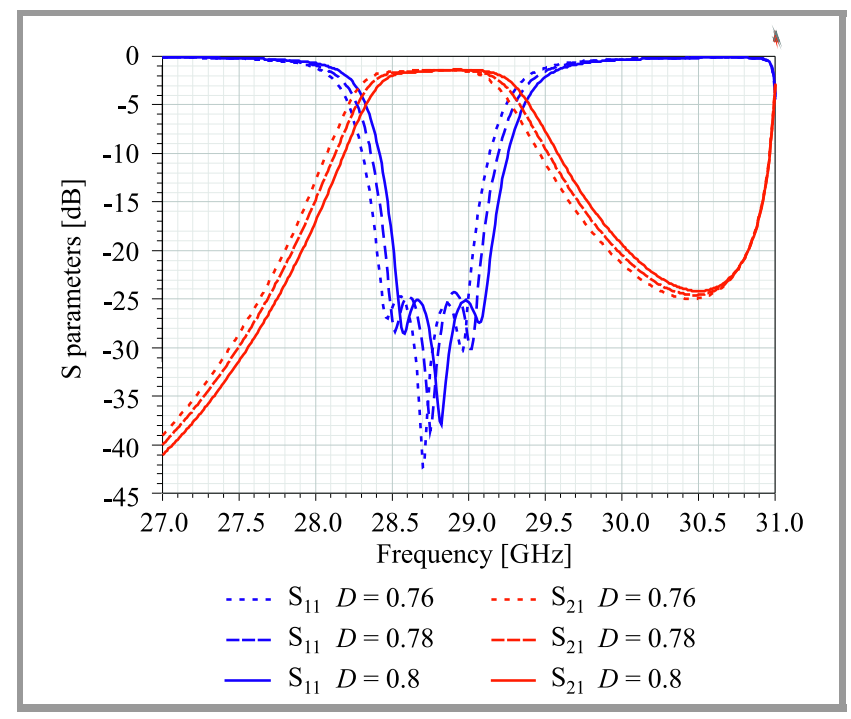

Fig. 11. Variation of the $D$ diameter.

resonant frequency may be a function of both, with changes to the opening diameter and $\mathrm{W}_{\text {SIW }}$ width introduced.

\section{SIW Band-pass Filter Based DGS Technique}

In the next step, the proposed model has been modified by implementing the defected ground structure (DGS), as shown in Fig. 12. The DGS technique is implemented through three-slot forms at the ground's upper surface, in

(a)

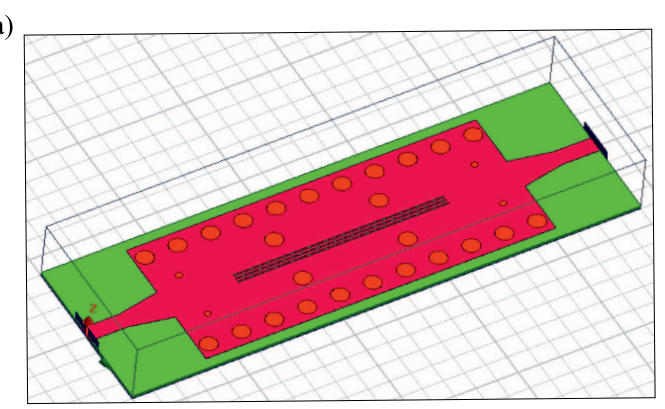

(b)

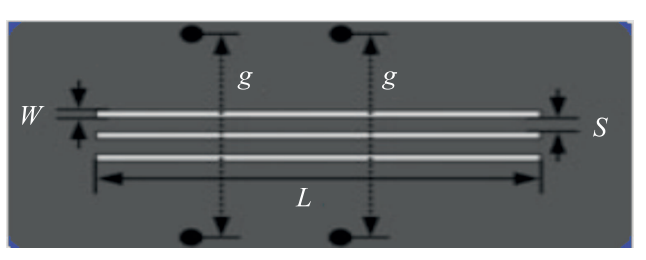

Fig. 12. Topology of the SIW band-pass filter using the DGS technique: (a) HFSS model, (b) slot geometry.

Table 4

Slot form dimensions

\begin{tabular}{|c|c|c|c|}
\hline Parameter & Value $[\mathrm{mm}]$ & Parameter & Value $[\mathrm{mm}]$ \\
\hline \hline$W$ & 0.1 & $g$ & 2.50 \\
\hline$S$ & 0.1 & $L$ & 9.95 \\
\hline
\end{tabular}

order to obtain a very low profile for a passband of $4 \mathrm{GHz}$ and center frequency of $27 \mathrm{GHz}$. Table 4 presents the parameters of the implemented slots described in Fig. 12.

Simulation results presented in Fig. 13 show that the response of the new filter is improved, as the low return loss equals $27 \mathrm{~dB}$ and good transmission with $1 \mathrm{~dB}$ for a central frequency of approximately $29 \mathrm{GHz}$ and a $4.12 \%$ fractional bandwidth.

Distribution of E field does not change when implementing slots at the ground Fig. 14. This demonstrates that the DGS technique allows to enhance the filter's response without affecting field distribution. In order to validate the two SIW filter models proposed above, a comparison with paper [11] using shunt inductive posts is performed

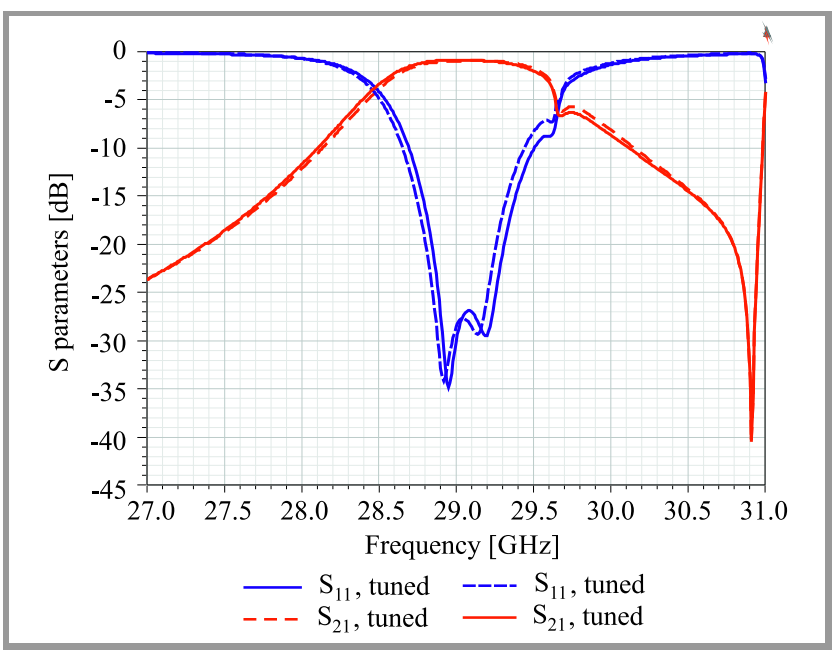

Fig. 13. S parameters of the SIW band-pass iris shunt inductive filter based on DGS.

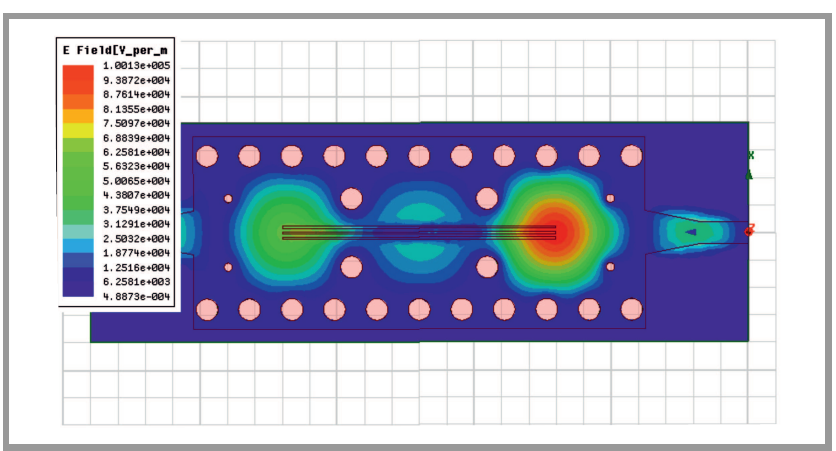

Fig. 14. Simulated E field distribution obtained by SIW DGS band-pass filter.

Table 5

EM frequency response comparison

\begin{tabular}{|c|c|c|c|}
\hline Reference & $\begin{array}{c}\text { FBW } \\
\text { and F0 }\end{array}$ & $\begin{array}{c}\text { Insertion loss } \\
{[\mathrm{dB}]}\end{array}$ & $\begin{array}{c}\text { Return loss } \\
{[\mathrm{dB}]}\end{array}$ \\
\hline \hline $\begin{array}{c}\text { [11], filter using } \\
\text { inductive shunt posts }\end{array}$ & $\begin{array}{c}3.87 \%, \\
29.18\end{array}$ & 1.5 & 15 \\
\hline $\begin{array}{c}\text { First model - SIW iris } \\
\text { inductive shunt filter }\end{array}$ & $\begin{array}{c}3.64 \%, \\
28.82\end{array}$ & 1.5 & 25 \\
\hline $\begin{array}{c}\text { Second model - SIW } \\
\text { based DGS filter }\end{array}$ & $\begin{array}{c}4.12 \%, \\
29.09\end{array}$ & 1.1 & 27 \\
\hline
\end{tabular}


for the same filter order and passband of between 28.35 and $29.75 \mathrm{GHz}$, as presented in Table 5 .

The proposed filter designs offer good electromagnetic performance in the Ka band. They are characterized by a low return loss and good transmission compared with the designs presented in other studies that validate both the accuracy of the modeling method relied upon and the efficiency of the selected EM simulator.

\section{Conclusion}

This paper presents two novel topologies of a third order band-pass iris shunt inductive filter using the SIW technology and the DGS technique. Both filters use the coupling matrix method which turns out to be a very useful tool for direct filter synthesis. Analytical formulas are used to calculate the filter's different geometric parameters and efficient optimization techniques are employed to reduce the effective dielectric permittivity on certain waveguide sections in order to achieve high performance demonstrated by a low reflection coefficient and a good transmission coefficient for the entire passband. Filters of this type are designed for use used in the Ka frequency band that is very suitable for satellite communications.

\section{References}

[1] I. Ohta, K. Toda, M. Kishihara, and T. Kawai, "Design of cruciform substrate-integrated waveguide hybrids based on H-plane planar circuit approach", in Proc. of Asia-Pacific Microw. Conf., Bangkok, Thailand, 2007, pp. 683-686 (DOI: 10.1109/APMC.2007.4554871).

[2] M. Bozzi, L. Perregrini, K. Wu, and P. Arcioni, "Current and future research trends in substrate integrated waveguide technology", Radio Engin., vol. 18, no. 2, 2009 [Online]. Available: https://www.radioeng.cz/fulltexts/2009/09_02_201_209.pdf

[3] A. Coves et al., "A novel passband filter based on a periodically drilled SIW Structure", Radio Sci., vol. 51, no. 4, pp. 328-336, 2016 (DOI: 10.1002/2015RS005874).

[4] L. Silvestri et al., "Modeling and implementation of perforated SIW filters", in Proc. IEEE MTT-S Int. Conf. on Numer. Electromag. and Multiphys. Model. and Optimiz. NEMO 2016, Beijing, China, 2016, pp. 209-210 (DOI: 10.1109/NEMO.2016.7561668).

[5] D. Dealandes and K. Wu, "Single-substrate integration techniques for planar circuits and waveguide filters", IEEE Trans. Microw. Theory Techn., vol. 51, no. 2, 593-596, 2003 (DOI: 10.1109/TMTT.2002.807820).

[6] S. Moscato, R. Moro, M. Pasian, M. Bozzi, and L. Perregrini, "Twomaterial ridge substrate integrated waveguide for ultra-wideband applications", IEEE Trans. Microw. Theory Techn., vol. 63, no. 10, pp. 3175-3182, 2015 (DOI: 10.1109/TMTT.2015.2461612).

[7] R. J. Cameron, "Advanced coupling matrix synthesis techniques for microwave filters", IEEE Trans. on Microw. Theory and Techn., vol. 51, no. 1, 2003 (DOI: 10.1109/TMTT.2002.806937).

[8] R. Bouhmidi, B. Bouras, and M. Chetioui, "Multi-ports extraction technique for microwave passband filter optimization", Int. J. of Microw. and Opt. Technol. (IJMOT), vol. 14, no. 6, pp. 431-439, 2019 [Online]. Available: https://ijmot.com/VOL-14-NO-6.aspx

[9] X.-P. Chen and K. Wu, "Substrate integrated waveguide filter: Basic design rules and fundamental structure features", IEEE Microw. Mag., vol. 15, no. 5, pp. 108-116, 2014 (DOI: 10.1109/MMM.2014.2321263).

[10] F. Xu and K. Wu, "Guided-wave and leakage characteristics of substrate integrated waveguide", IEEE Trans. on Microw. Theory and Techn., vol. 53, no. 1, pp. 66-73, 2005 (DOI: 10.1109/TMTT.2004.839303).
[11] F. Parment, A. Ghiotto, T. P. Vuong, J. M. Duchamp, and K. Wu, "Air-filled substrate integrated waveguide for low-loss and high powerhandling millimeter-wave substrate integrated circuits", IEEE Trans. Microw. Theory Techn., vol. 63, no. 4, pp. 1228-1238, 2015 (DOI: 10.1109/TMTT.2015.2408593).

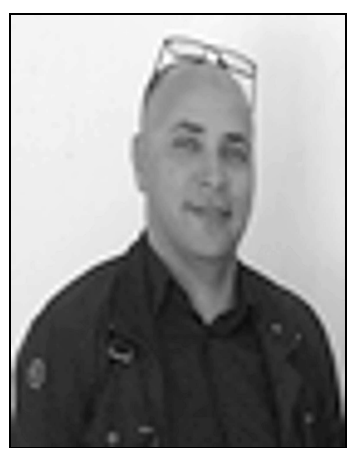

Mehdi Damou received his Ph.D. in Telecommunications from Abu Bakr Belkaid University of Tlemcen, Algeria, in 2018. He is a lecturer and the Head of the Electronics Department at Dr. Tahar Moulay University of Saida, Algeria. His research interests include microwave and RF devices and components. He is working on developing antennas designs and microwave filters based on SIW technologies and efficient EM modeling techniques.

(D) https://orcid.org/0000-0003-4448-3318

E-mail: bouazzamehdi@yahoo.fr

Laboratory of Technologies of Communications

Dr. Tahar Moulay University of Saida

Saida, Algeria

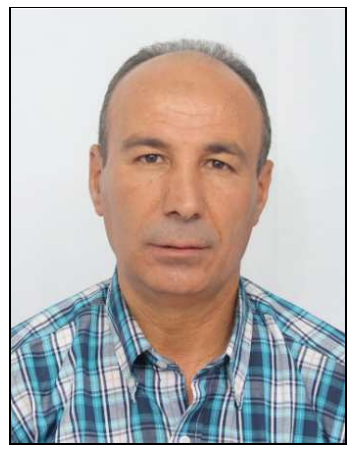

Yassine Benallou received his Ph.D. in Electronics from Djillali Liabes University of Sidi Bel Abbes, Algeria, in 2014. $\mathrm{He}$ is a lecturer at the Electronics Department of Dr. Tahar Moulay University of Saida, Algeria and has been a senior member at the Laboratory of Technologies of communications since its establishment. His academic research focuses on different fields, including modeling and designing of electronic circuits and systems, optimizing passive EM components and characterizing new materials for biomedical and renewable applications.

E-mail: benallou06@yahoo.fr Laboratory of Technologies of Communications Dr. Tahar Moulay University of Saida Saida, Algeria

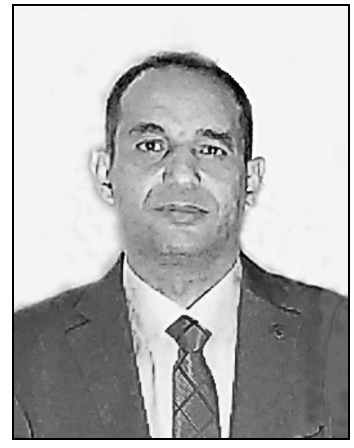

Mohammed Chetioui received his Ph.D. in Telecommunications from Abu Bakr Belkaid University of Tlemcen, Algeria, in 2018. Since then, he has been a lecturer at the Electronics Department of Dr. Tahar Moulay University of Saida, Algeria. His research interests include digital communications, signal processing, microwave circuits 
and RF systems. He is working on designing passive/active microwave filters based on the microstrip technology and accurate optimizations.

E-mail: chetioui.mohammed@yahoo.fr

Laboratory of Telecommunications

Abu Bakr Belkaid University of Tlemcen

Tlemcen, Algeria

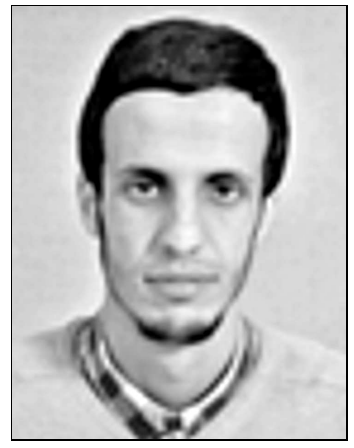

Abdelhakim Boudkhil received his Ph.D. in in Electronics from Abu Bakr Belkaid University of Tlemcen, Algeria, in 2018. He is an Assistant Professor at the Electronics Department at Dr. Tahar Moulay University of Saida, Algeria. His research experience concerns several fields, including digital, optical, microwave, and RF communication systems. His research interests focus more on optimizing and developing antennas based on integrated technologies and advanced techniques.
E-mail: boudkhil.abdelhakim@yahoo.fr Laboratory of Telecommunications Abu Bakr Belkaid University of Tlemcen Tlemcen, Algeria

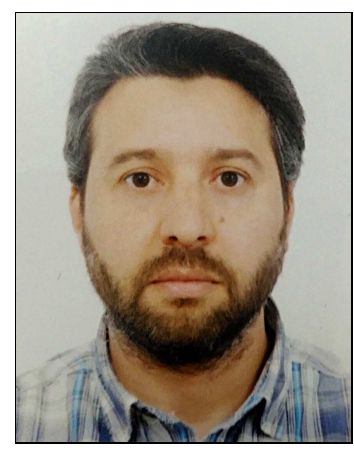

Redouane Berber received his M.Sc. in Electronics from Dr. Tahar Moulay University of Saida, Algeria, in 2008. $\mathrm{He}$ is an Assistant Professor and a deputy head at the Electronics Department at Dr. Tahar Moulay University of Saida, Algeria. His research interests focus specifically on electronic components and systems, as well as on optical communications and networking. $\mathrm{He}$ is working on investigating different multiple access techniques.

E-mail: red1ber@gmail.com

Laboratory of Technologies of Communications

Dr. Tahar Moulay University of Saida

Saida, Algeria 